\title{
Local Physics and Star Formation in Galaxies
}

\author{
Adam K. Leroy, Frank Bigiel, Annie Hughes, Eva Schinnerer, \\ Antonio Usero and the PAWS, EMPIRE, and HERACLES Dense \\ Gas Collaborations
}

\begin{abstract}
A next main step in understanding star formation is to link the sharp but narrow view of Galactic molecular cloud studies to the wider context accessed by less detail by extragalactic work. In this proceeding, we discuss how new technology and large programs at millimeter wavelengths are improving our ability to access physical conditions in the interstellar medium (ISM) of other galaxies. We highlight results from the multi-line survey of Usero et al. (2015), which measured density sensitive lines across nearby galaxy disks, and two new mapping studies of M51: the high resolution Plateau de Bure Arcsecond Whirlpool Survey (PAWS) and the EMPIRE multi-line mapping survey. These results argue for a context-dependent role for gas density in star formation; that is, gas at a particular density does not appear to form stars in a universal way. They also demonstrate the influence of cloud-scale conditions, especially surface density and the velocity dispersion, in setting the small-scale density distribution and highlight gravitational boundedness as a main driver of the ability of gas to form stars. Beyond these specific results, we argue that ability to gauge detailed physical conditions in the star-forming gas of other galaxies promises major advances that will help unify the fields of Galactic and extragalactic star formation in the next few years.
\end{abstract}

\section{Introduction}

The last decade has seen major strides in how we understand star formation on the scale of galaxies and on the scale of individual molecular clouds. A key next challenge is to connect these two views and better unify the fields of Galactic and extra-galactic star formation. The main obstacle to this has been the dramatically different scales accessed by studies targeting nearby Milky Way clouds and those focused on other galaxies. The former heavily resolve individual clouds, so that cloud substructure and local conditions like gas volume density and the sonic Mach number can be directly compared to the ability of gas to form stars (e.g., Kainulainen et al. 2009; Lada et al. 2012; André et al. 2014). Extragalactic observations have, so far, struggled to access these conditions in a systematic way. In exchange for this more "blurry" view, however, they provide an unparalleled accounting of gas and star formation across the universe and the galaxy population (e.g., Young et al. 1995; Saintonge et al. 2011, Utomo et al., in prep. and in this volume). Observations that resolve galaxies but not clouds also provide our best tool, so far, to link the process of star formation to galactic structure (e.g., Leroy et al. 2008; Bigiel et al. 2008; Schruba et al. 2011; Leroy et al. 2013). Thus, extragalactic observations yield the best answer to date for "How does the process of star formation depend on the larger galactic context?"

This situation is improving rapidly. Over the last few years, Galactic studies have improved in their perspective, with wide field, high resolution maps of interstellar medium (ISM) structure from Herschel (e.g., Schneider et al. 2015) and the contrast between the Solar Neighborhood and the Central Molecular Zone offering prime examples (Longmore 

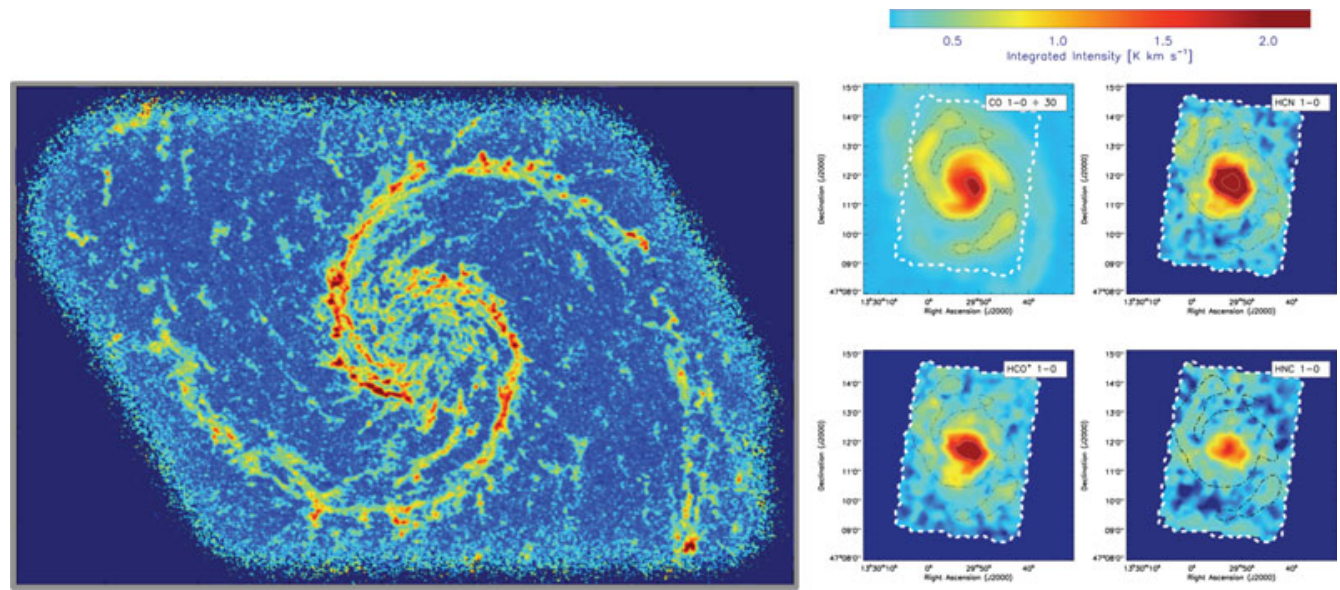

Figure 1. Resolution and spectroscopic mapping. (left) The PAWS view of the inner region of M51, from which we know the surface density, velocity dispersion, and boundedness of gas at $40 \mathrm{pc}$ resolution. (right) The EMPIRE spectral mapping survey's view of M51 in the density-sensitive line $\mathrm{HCN}, \mathrm{HCO}^{+}$, and $\mathrm{HNC}$. Combining these views give new access to the physical state of the gas in galaxies. Color versions of the figures are available online.)

et al. 2013; Kauffmann et al. 2013). Many of these efforts are discussed elsewhere in these proceedings. In this contribution, we emphasize improvements from the other direction: thanks to a mixture of new technology and major commitments from existing facilities, extragalactic observations increasingly access some of the small-scale physics previously restricted to studies of the Milky Way and the nearest galaxies in the Local Group.

We demonstrate the bright future for this approach using results from new spectroscopic surveys (Usero et al. 2015, and "EMPIRE" — Bigiel et al. in prep.) and the PdBI Arcsecond Whirlpool Survey (Schinnerer et al. 2013; Pety et al. 2013). These demonstrate a changing ratio of star formation rate and high critical density line emission, which we argue to be best explained by a context-dependent role for density in star formation. In M51, they also show a close link between the "cloud scale" ( $~ 40$ pc) ISM structure traced by PAWS and the density distribution traced by the EMPIRE spectroscopy. We also show that in PAWS, gravitational boundedness appears to be the structural property most closely associated with star formation. The new results comparing these approaches for M51 are presented in Leroy et al. (in prep.).

\section{Two Old Tools That Are New Again: Resolution and Spectroscopy}

Based on both theoretical and observational work, local conditions in the ISM are believed to affect its ability to form stars. For purposes this proceeding, there are two main families of ISM properties expected to drive star formation in gas. First, the gas volume density is considered pivotal by almost all theories of star formation. Turbulent models (e.g., Krumholz \& McKee 2005) consider the free fall time, $\tau_{\mathrm{ff}} \propto \rho^{-0.5}$, the governing timescale for star formation. As a result, in these models, the normalized rate of star formation, SFR-per-unit-gas, tracks the gas volume density closely. Another family of models, mostly motivated by observations, posit a threshold volume density above which star formation proceeds in a more or less universal way (e.g., Gao \& Solomon 2004; Heiderman et al. 2010; Lada et al. 2012; Evans et al. 2014). In this case, the main determinant of the ability of gas to form stars is the fraction of gas mass above this threshold, $f_{\text {dense }}=M_{\text {dense }} / M_{\text {gas }}$. 
The local gas volume density is primarily accessible to extragalactic observers via multiline mm-wave spectroscopy. A main result mapping Galactic molecular clouds is that the dense, star-forming gas is concentrated into very small structures, $\sim 0.1 \mathrm{pc}$-wide filaments and $\sim$ pc scale dense clumps. Even with the Atacama Large Millimeter/submillimeter Array, such structures are accessible to direct imaging in other galaxies only under special circumstances and with great effort. Fortunately, the mm-wave band contains transitions from molecules with a wide range of dipole moments and so a wide range of density sensitivities. The distribution of gas density within a beam can be accessed by observing both molecules like ${ }^{12} \mathrm{CO}$, which — after accounting for optical depth — trace almost all molecular with molecules like $\mathrm{HCN}$, which is effectively excited at $\mathrm{H}_{2}$ densities $\gtrsim$ $10^{6} \mathrm{~cm}^{-3}$ (before accounting for optical depth).

The power of combining lines with different density sensitivities has long been recognized and has been exploited in classic work by Gao \& Solomon (2004). Until recently, the faintness of high critical density lines (often $\sim 1 / 30$ the intensity of CO or less; see Usero et al. 2015) has limited their widespread deployment to understand the density distribution in normal galaxies. With the improved sensitivity and bandwidth of new receivers (like EMIR on the IRAM 30-m) and new facilities (especially ALMA) this situation is changing. Usero et al. (2015) used EMIR to make the first systematic survey of density-sensitive lines spanning the disks of normal star-forming galaxies, a complement to the active galaxies and center-dominated measurements of Gao \& Solomon (2004). The IRAM large program EMPIRE (P.I. F. Bigiel) is now mapping lines with a suite of critical densities across the entire disk of 9 galaxies, beginning with a prototype program mapping several lines across the whole disk of M51 (Bigiel et al., in prep. and this volume; see also Chen et al. 2015). This is the first full-galaxy data set covering lines with a wide range of critical densities and has the advantage of overlapping the high resolution view of PAWS.

In turbulent models, the cloud-scale structure of the ISM affects the ability of gas at a given density to form stars (i.e., the star formation efficiency per free-fall time; e.g., Federrath \& Klessen 2012). The mean density of a cloud, related to the cloud-scale surface density, $\Sigma$, relates to the free-fall time on the scale of the whole cloud. For a fixed temperature, the cloud-scale velocity dispersion, $\sigma$, is a close cognate of the turbulent Mach number, which affects the width of the density distribution. The combination of these two parameters tracks the gravitational boundedness of the cloud; this is often expressed as the virial parameter, $\alpha_{\text {vir }}=0.5 \mathrm{KE} / U \mathrm{E}$. In Leroy et al. (in prep.) we argue that in full-fidelity, high resolution imaging like PAWS the beamwise quantity $\Sigma / \sigma^{2}$, which we call pixelwise "boundedness," captures the physics of the virial parameter.

Unlike the very small-scale distribution of densities, these cloud-scale quantities are potentially accessible from direct imaging using ALMA or NOEMA for a large set of galaxies. Arcsecond resolution CO imaging can readily resolve the structure of the ISM at $\sim 50$ pc scales out to the Virgo cluster. The PAWS survey serves a clear demonstration of the power of this approach, showing clear variations of cloud properties with environment in the galaxy (Hughes et al. 2013; Colombo et al. 2014). While a few times 10 pc resolution only marginally resolves a Galactic cloud, the physical link between gas structure at this scale and star formation is much larger than for ISM properties measured over beams $\sim \mathrm{kpc}$ in area or more. To some approximation, one can think of this high resolution, high sensitivity, full-fidelity CO mapping as providing galaxy-wide maps of the initial conditions for star formation in molecular clouds.

Connecting these approaches, it is possible to relate cloud-scale properties to the small scale density distribution and to link both to the ability of gas to form stars. As the prototype for all of these types of observations, M51 is a natural first target for such 

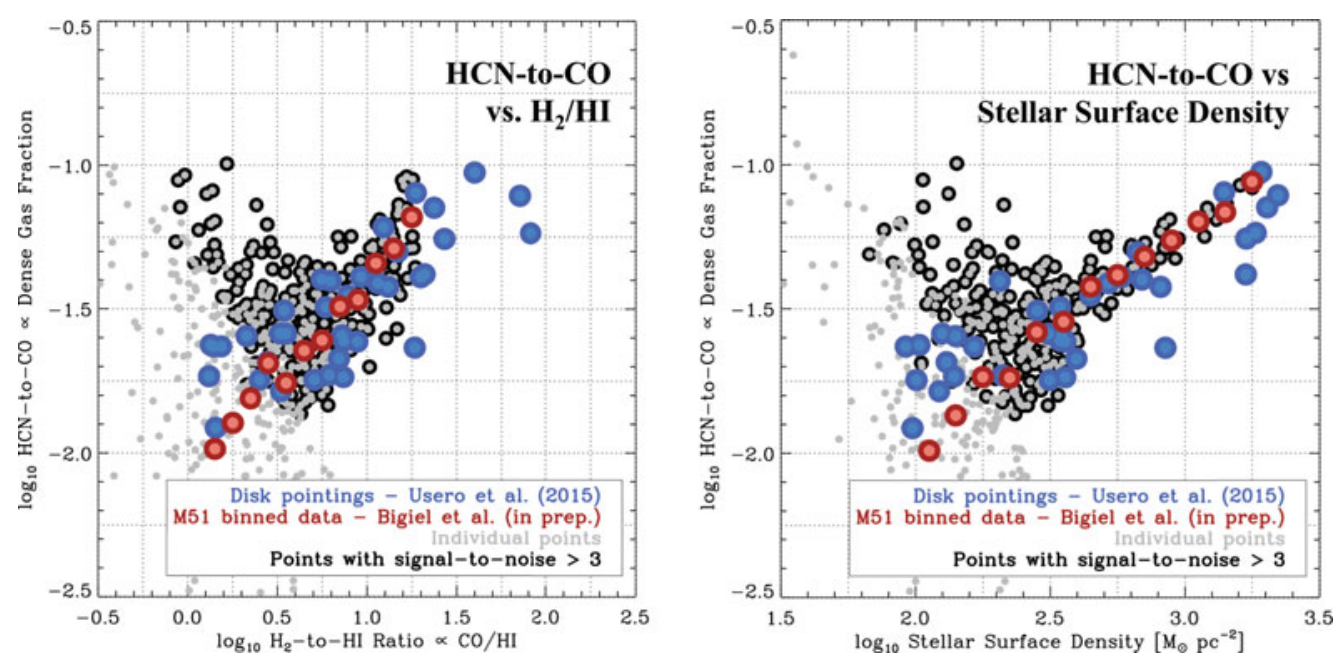

Figure 2. HCN-to-CO ratio, indicative of the fraction of gas mass as a function of the molecular-to-atomic gas ratio (left) and stellar surface density (right), which both track average ISM pressure or density. Blue points show individual disk pointings from Usero et al. (2015), gray points show individual lines of sight in M51 from Bigiel et al. (in prep.), with black outlines indicating where signal-to-noise is greater than 3 . Red points show the binned trend for M51. The fraction of mass in a dense phase increases with increasing surface density, molecular fraction, and ISM pressure.

studies. Results for individual approaches targeting M51 have appeared in Meidt et al. (2013); Usero et al. (2015, Bigiel et al. in prep.); In Leroy et al. (in prep.), we combine these views.

\section{A Context-Dependent Role for Gas Density}

In Usero et al. (2015) and Bigiel et al. (in prep.), we measure the distribution of lines with varying critical densities across the disks of spiral galaxies - the whole disk of M51 in Bigiel et al. (in prep.) and pointings at a range of radii in 30 disk galaxies in Usero et al. (2015). Here we highlight results for the HCN (1-0) transition but both surveys measure several transitions. Following Gao \& Solomon (2004), HCN (1-0) has been commonly identified as tracing specifically star-forming, high density gas. A nearly fixed relationship between HCN (1-0) and infrared luminosity has been linked to results from the Solar Neighborhood to argue for an approximately universal role for gas above this threshold density in star formation. Matched measurements of $\mathrm{CO}, \mathrm{HCN}$, and recent star formation (mostly traced here by IR luminosity) across galaxy disks thus allow us to ask two key questions: "What environmental factors regulate the fraction of dense gas mass?" and "Does high density gas always form stars at approximately the same rate?" Perhaps surprisingly, the whole-disk map of M51 by Bigiel et al. (in prep.) and the disk pointing survey of Usero et al. (2015) yield consistent results for both questions.

In Usero et al. (2015), we show that the fraction of dense gas, traced by the HCN-to-CO ratio (thanks to their very different effective densities), varies strongly across galaxies. The sense of the variation is that a higher fraction of gas is dense in the inner, high surface density parts of galaxies where most of the gas is molecular. Though neither relationship is necessarily causal, this behavior is well-encapsulated by a correlation of the ratio $\mathrm{HCN}-$ to-CO, tracing the dense gas mass fraction, with stellar surface density, $\Sigma_{\star}$, and the molecular-to-atomic ratio, $\Sigma_{\mathrm{H} 2} / \Sigma_{\mathrm{Hi}}$. In Bigiel et al. (in prep.), we show that 
M51 obeys the same behavior, with individual $\approx 1 \mathrm{kpc}$ resolution lines of sight exhibiting a correlation that overlaps the pointings from $\approx 30$ disk galaxies in Usero et al. (2015). Thus, in the high surface density, highly molecular parts of galaxies, where the ISM pressure and mean ISM density is also high, a larger fraction of the molecular gas tends to be in a dense phase. In Leroy et al. (in prep.), we show that in M51, the full pattern of line ratios from ${ }^{12} \mathrm{CO}(1-0)$ through $\mathrm{HCN}$ (1-0) are consistent with an interpretation in which this changing $\mathrm{HCN}$-to-CO ratio indeed reflects changes in the mean ISM density.

Finding more dense gas in the denser parts of galaxies is expected, though not a given (molecular gas might simply have a higher filling factor in these locales). The more surprising result of these surveys of density-sensitive lines is that the link between dense gas and star formation seems to vary with environment. Specifically, in Usero et al. (2015) we show that the ratio of SFR-to-HCN, a close cognate of the rate at which dense gas forms stars, anti-correlates with the stellar surface density and molecular fraction. That is, the deeper the stellar potential well and the more molecular the ISM, the less efficient dense gas is at forming stars. This result is reinforced by results from full-disk mapping in M51. This agrees with the sense of results contrasting the Solar Neighborhood with the Galactic Center (e.g., Longmore et al. 2013), but strongly disagrees with the family of results that posit a universal process of star formation in the dense gas traced by HCN (e.g., Gao \& Solomon 2004; Lada et al. 2012).

The natural explanation for this varying IR-to-HCN ratio is a context-dependent role for density in star formation. As a simple example, if star formation is driven by a density contrast with a marginally bound background cloud, then the density of the background cloud will define what qualifies as "dense" gas for purposes of star formation. Put another way, HCN may trace immediately star-forming gas in a region where the mean density of cold clouds is $\langle n\rangle \sim 10^{3} \mathrm{~cm}^{-3}$. But in a region with higher average density, say $\langle n\rangle \sim 10^{4} \mathrm{~cm}^{-3}$, HCN will trace a larger fraction of each cloud, including potentially non self-gravitating, non star-forming gas. Versions of this scenario have been put forward by Krumholz \& Thompson (2007), Narayanan et al. (2008), and are nicely synthesized in Federrath \& Klessen (2012).

Our observations of an IR-to-HCN ratio that decreases when the ISM appears to become more dense on average provide strong observational support to this theoretical scenario. They argue that the role of gas at a particular volume density depends on context, especially the mean density and gravitational boundedness of the interstellar medium on larger (cloud) scales.

\section{A Link Between Cloud-Scale ISM Structure and Star Formation}

Results from multi-line spectroscopy thus suggest an important role for cloud-scale ISM properties in the ability of gas to form stars. Thanks to PAWS, we know the local surface density $(\Sigma)$, velocity dispersion $(\sigma)$, and boundedness $\left(\Sigma / \sigma^{2} \propto \alpha_{\text {vir }}^{-1}\right)$ of the molecular gas at $40 \mathrm{pc}$ resolution. In Leroy et al. (in prep.), we convolve these local measurements to the $\approx 1 \mathrm{kpc}$ resolution of the infrared data and multi-line spectroscopy. During this convolution, we weight the small-scale properties by the local CO luminosity so that we measure the luminosity-weighted property within the beam; this is closely related to a mass weighting and is only possible starting from the high resolution PAWS data. Thus $\Sigma$ measured in this way over a kpc is effectively the average surface density at 40 -pc from which a CO photon arises in the PAWS map; $\sigma$ and $\Sigma / \sigma^{2}$ are analogous.

Following the discussion in the previous two parts, PAWS offers us the opportunity to ask how cloud-scale ISM structure affects the ability of gas to form stars, here traced by the ratio IR-to-CO, and the fraction of gas in a dense phase. The figures here (from 

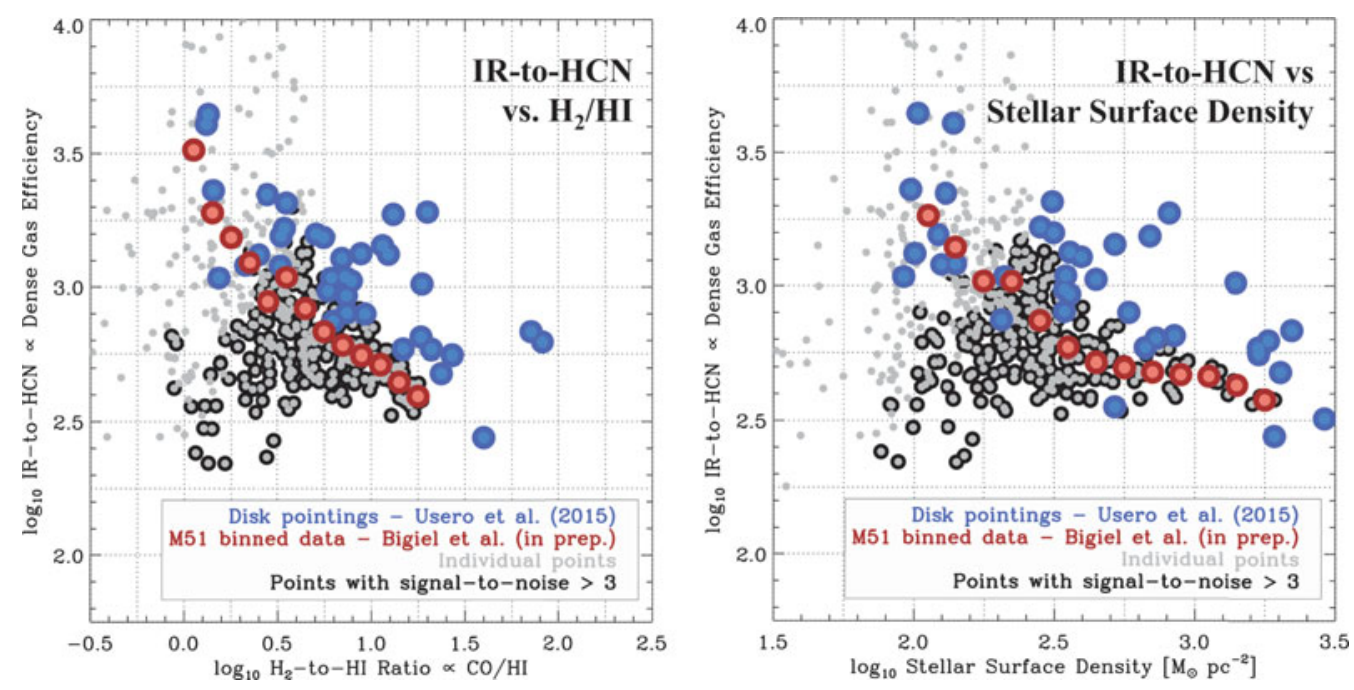

Figure 3. As Figure 1 but now showing the ratio of star formation traced by IR emission to dense gas traced by HCN emission as a function (left) and stellar surface density (right), which both track average ISM pressure or density. Blue points show individual disk pointings from Usero et al. (2015), gray points show individual lines of sight in M51 from Bigiel et al. (in prep.), with black outlines indicating where signal-to-noise is greater than 3 . Red points show the binned trend for M51. Contrary to hypotheses based on the Solar Neighborhood, the star formation per unit dense gas varies systematically in galaxies. The sense is that in the denser parts of galaxies, the star formation per unit dense gas is lower. This is consistent with a context-dependent role for gas density in star formation, in which contrast with the background matters as much as the absolute value of the density.

Leroy et al. in prep) show that a high cloud scale surface density and a high velocity dispersion do correspond to a larger fraction of gas in a dense, star-forming phase. But the relationship of these quantities to the ability of all of the gas to form stars, tracked by IR-to-CO, is less clear, with both high and low star formation efficiencies at high values of $\Sigma$ and $\sigma$. Instead, the apparent boundedness of the gas, traced by $\Sigma / \sigma^{2}$ offers our best cloud-scale predictor for how well gas forms stars.

These results agree well with the picture outlines in the previous section: gas volume density exhibits a context dependent role in star formation. There will be a higher fraction of dense gas in regions with high average (cloud-scale) densities. But this gas also tends to show a lower ratio of recent star formation rate to dense gas (IR-to-HCN). A natural explanation for this is that the HCN emitting gas in these regions traces not only the highly self-gravitating tail but also a larger part of the non-self-gravitating density distribution. Other considerations, especially a variable optical depth of the dense gas tracers (Jesus-Jimenez in prep.) may also help explain this effect, but we argue that the pattern of line ratios and the visible change in cloud-scale surface density is consistent with this interpretation.

Regardless of interpretation these results undermine a main set of arguments for models in which star formation proceeds in a universal way about some volume density. These models have invoked a universal scaling between HCN and tracers of recent star formation as a demonstration as the main extragalactic evidence that dense gas forms stars in a universal way (e.g., Gao \& Solomon 2004; Lada et al. 2012). Although there remains an broad correlation between between IR luminosity and HCN luminosity across many 

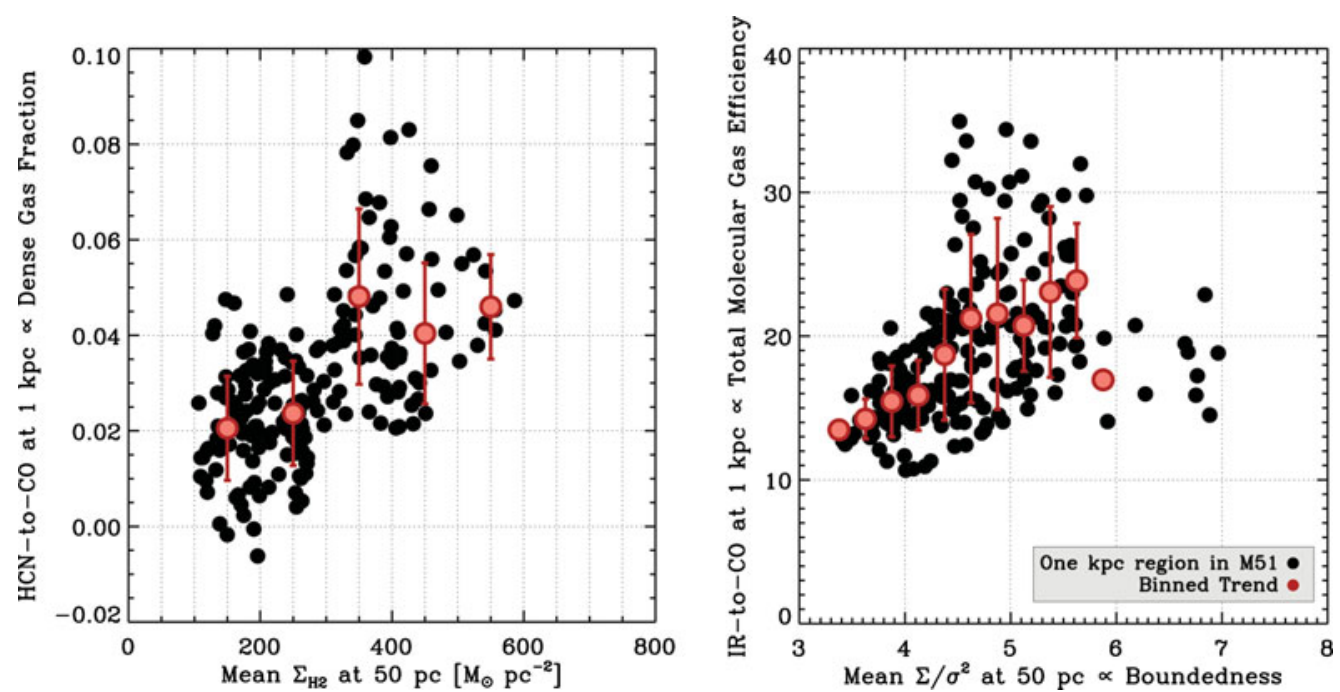

Figure 4. Link between cloud-scale (40 pc resolution) ISM structural properties measured from PAWS and the ability of the ISM to form stars at larger scales. (left) The fraction of dense gas mass correlates with the mass weighted cloud-scale surface density in a beam. (right) The overall ability of gas to form stars correlates with the mass-weighted gravitational boundedness in a beam. The link between the overall ability of the molecular gas to form stars, the distribution of small-scale densities traced by spectroscopy, and the cloud-scale structural properties show how galaxy-scale processes connect to the small-scale physics of star formation, a link that promises to be productively explored with ALMA, NOEMA, the GBT, and similar facilities in coming years.

decades (Gao \& Solomon 2004; Wu et al. 2005), in detail the star formation rate per unit $\mathrm{HCN}$ shows real physical variations.

\section{Outlook}

Beyond the specific results highlighted above, a main goal of this proceeding is to sound an optimistic note regarding studies of star formation in the next few years. The ability of telescopes including ALMA and NOEMA to resolve cloud-scale ISM structure opens a new window to link galaxy-scale processes to the immediate sites of star formation. The sensitivity of facilities including ALMA, NOEMA, the Green Bank Telescope (now a mmwave spectroscopic facility), and the IRAM 30-m telescope also at last allows multi-line mm-wave spectroscopy to be deployed as a survey tool, not restricted to individual case studies or the most extreme systems. This promises a window into small scale conditions including gas volume density, which is commonly viewed as a governing parameter for the ability of gas to form stars. Here we show that the large progress may be expected from combining these two views, and thus measuring how galactic structure sets cloud scale conditions, how these drive small-scale physics, and how they all relate to star formation in clouds.

\section{References}

André, P., Di Francesco, J., Ward-Thompson, D., Inutsuka, S.-I., Pudritz, R. E., \& Pineda, J. E. 2014, Protostars and Planets VI, 27

Bigiel, F., Leroy, A., Walter, F., Brinks, E., de Blok, W. J. G., Madore, B., \& Thornley, M. D. $2008, A J, 136,2846$ 
Chen, H., Gao, Y., Braine, J., \& Gu, Q. 2015, ApJ, 810, 140

Colombo, D., Hughes, A., Schinnerer, E., Meidt, S. E., Leroy, A. K., Pety, J., Dobbs, C. L., Garcia-Burillo, S., Dumas, G., Thompson, T. A., Schuster, K. F., \& Kramer, C. 2014, ArXiv e-prints

Evans, II, N. J., Heiderman, A., \& Vutisalchavakul, N. 2014, ApJ, 782, 114

Federrath, C. \& Klessen, R. S. 2012, ApJ, 761, 156

Gao, Y. \& Solomon, P. M. 2004, ApJ, 606, 271

Heiderman, A., Evans, II, N. J., Allen, L. E., Huard, T., \& Heyer, M. 2010, ApJ, 723, 1019

Hughes, A., Meidt, S. E., Colombo, D., Schinnerer, E., Pety, J., Leroy, A. K., Dobbs, C. L., García-Burillo, S., Thompson, T. A., Dumas, G., Schuster, K. F., \& Kramer, C. 2013, ApJ, 779,46

Kainulainen, J., Beuther, H., Henning, T., \& Plume, R. 2009, AAP, 508, L35

Kauffmann, J., Pillai, T., \& Zhang, Q. 2013, it ApJL, 765, L35

Krumholz, M. R. \& McKee, C. F. 2005, ApJ, 630, 250

Krumholz, M. R. \& Thompson, T. A. 2007, ApJ, 669, 289

Lada, C. J., Forbrich, J., Lombardi, M., \& Alves, J. F. 2012, ApJ, 745, 190

Leroy, A. K., Walter, F., Brinks, E., Bigiel, F., de Blok, W. J. G., Madore, B., \& Thornley, M. D. 2008, $A J, 136,2782$

Leroy, A. K., Walter, F., Sandstrom, K., Schruba, A., Munoz-Mateos, J.-C., Bigiel, F., Bolatto, A., Brinks, E., de Blok, W. J. G., Meidt, S., Rix, H.-W., Rosolowsky, E., Schinnerer, E., Schuster, K.-F., \& Usero, A. 2013, ArXiv e-prints

Longmore, S. N., Bally, J., Testi, L., Purcell, C. R., Walsh, A. J., Bressert, E., Pestalozzi, M., Molinari, S., Ott, J., Cortese, L., Battersby, C., Murray, N., Lee, E., Kruijssen, J. M. D., Schisano, E., \& Elia, D. 2013, MNRAS, 429, 987

Meidt, S. E., Schinnerer, E., García-Burillo, S., Hughes, A., Colombo, D., Pety, J., Dobbs, C. L., Schuster, K. F., Kramer, C., Leroy, A. K., Dumas, G., \& Thompson, T. A. 2013, ApJ, 779, 45

Narayanan, D., Cox, T. J., Shirley, Y., Davé, R., Hernquist, L., \& Walker, C. K. 2008, ApJ, 684,996

Pety, J., Schinnerer, E., Leroy, A. K., Hughes, A., Meidt, S. E., Colombo, D., Dumas, G., García-Burillo, S., Schuster, K. F., Kramer, C., Dobbs, C. L., \& Thompson, T. A. 2013, ApJ, 779, 43

Saintonge, A., Kauffmann, G., Wang, J., Kramer, C., Tacconi, L. J., Buchbender, C., Catinella, B., Graciá-Carpio, J., Cortese, L., Fabello, S., Fu, J., Genzel, R., Giovanelli, R., Guo, Q., Haynes, M. P., Heckman, T. M., Krumholz, M. R., Lemonias, J., Li, C., Moran, S., Rodriguez-Fernandez, N., Schiminovich, D., Schuster, K., \& Sievers, A. 2011, MNRAS, 415, 61

Schinnerer, E., Meidt, S. E., Pety, J., Hughes, A., Colombo, D., García-Burillo, S., Schuster, K. F., Dumas, G., Dobbs, C. L., Leroy, A. K., Kramer, C., Thompson, T. A., \& Regan, M. W. 2013, ApJ, 779, 42

Schneider, N., Bontemps, S., Girichidis, P., Rayner, T., Motte, F., André, P., Russeil, D., Abergel, A., Anderson, L., Arzoumanian, D., Benedettini, M., Csengeri, T., Didelon, P., Di Francesco, J., Griffin, M., Hill, T., Klessen, R. S., Ossenkopf, V., Pezzuto, S., RiveraIngraham, A., Spinoglio, L., Tremblin, P., \& Zavagno, A. 2015, MNRAS, 453, L41

Schruba, A., Leroy, A. K., Walter, F., Bigiel, F., Brinks, E., de Blok, W. J. G., Dumas, G., Kramer, C., Rosolowsky, E., Sandstrom, K., Schuster, K., Usero, A., Weiss, A., \& Wiesemeyer, H. 2011, AJ, 142, 37

Usero, A., Leroy, A. K., Walter, F., Schruba, A., García-Burillo, S., Sandstrom, K., Bigiel, F., Brinks, E., Kramer, C., Rosolowsky, E., Schuster, K.-F., \& de Blok, W. J. G. 2015, AJ, 150,115

Wu, J., Evans, II, N. J., Gao, Y., Solomon, P. M., Shirley, Y. L., \& Vanden Bout, P. A. 2005, it ApJL, 635, L173

Young, J. S., Xie, S., Tacconi, L., Knezek, P., Viscuso, P., Tacconi-Garman, L., Scoville, N., Schneider, S., Schloerb, F. P., Lord, S., Lesser, A., Kenney, J., Huang, Y.-L., Devereux, N., Claussen, M., Case, J., Carpenter, J., Berry, M., \& Allen, L. 1995, ApJS, 98, 219 